\title{
A Deterministic Model Of HIV Transmission Between Two Closed Patches Incorporating The Monod Equation
}

\author{
James Khobocha Mirgichan ${ }^{1 *}$ Cyrus Gitonga Ngari ${ }^{2}$ Stephen Karanja ${ }^{3}$ \\ 1* Department of Mathematics, School of Pure and Applied Sciences, Meru University of Science and \\ Technology-Kenya. Tel: +254722680624 \\ 2 Department of Mathematics, Computing and Information, School of Pure and Applied Sciences, University of \\ Embu-Kenya. Tel: +254711551568; E-mail: cyrus_ngari@yahoo.com \\ 3 Department of Mathematics, School of Pure and Applied Sciences, Meru University of Science and \\ Technology-Kenya. Tel: +254723520493; E-mail: stephenkaranja16@gmail.com \\ *E-mail of the corresponding Author: kaks.mirgichan@gmail.com
}

\begin{abstract}
Among other factors, migration has significantly contributed to the spread of HIV. Recent studies have revealed that new infections occur along major transport corridors and truck-drivers have overall higher prevalence rates of HIV and sexually transmitted infections than non-truck drivers' counterparts. Therefore, there exist a link between population mobility and HIV infection, as populations along transport corridors remain substantial contributors of new infections. This research work documents a deterministic model of the dynamics of HIV transmission between two closed patches that incorporates the Monod equation in migration with truck drivers being the agents of HIV transmission. Migration is considered as a social determinant to health and have a significant impact on healthrelated vulnerabilities and access to services. We assumed that susceptible individuals become infected via sexual intercourse with HIV infected truck drivers and all the infected individuals ultimately developed AIDS exponentially. The model also assumed that the patches have different infection and susceptibility rates. The patches basic reproduction number, $R_{0}$ was determined using the Next Generation Matrix. The results revealed that $R_{0}$ should be kept below unity to eradicate the transmission of the virus. The Disease-Free Equilibrium Point was obtained based on the signs of the Eigen values of the Jacobian matrix. In the absence, the Disease-Free Equilibrium Point is both Locally Asymptotically and Globally Asymptotically Stable. It was further proved that the model did not display Endemic Equilibrium Point under a special property for epidemic models. The model findings are vital in guiding health practitioners, governmental and non-governmental health agencies in the development of effective mitigation strategies to reduce the spread of HIV.
\end{abstract}

KEY WORDS: HIV/AIDS, Migration, Monod function, Basic reproduction number, Stability Analysis, Equilibria Points.

DOI: 10.7176/MTM/9-6-03

Publication date: June $30^{\text {th }} 2019$

\section{Introduction}

\subsection{Background of the study}

Human immunodeficiency virus (HIV) is a retrovirus that mainly infects CD4+ T-cells. The infected cell undergoes through three stages; asymptomatic (0-3yrs); Symptomatic (3-8yrs), AIDS (8yrs and above), based on the viral load and CD4+ counts. AIDS is Acquired Immunodeficiency Syndrome, that consist of a set of symptoms and illness caused by HIV. The immune system of an infected individual becomes too weak to fight further infections or illness. CD4+ T-cells produces new virions, which lead to more cell infection and viral production. Among the humans it's transmitted as a result of sexual intercourse between the susceptible individual and infected person. It's not transmitted through saliva, urine or sweat. HIV is found in vaginal fluid, semen, blood, anal fluids and breastmilk. AIDS has no cure but infected persons can enjoy better living if given support and treatment is taken correctly (Avert 2019). HIV continues to be a major global public health concern, having claimed more than 35 million lives so far despite various attempts to reduce this menace (WHO and UNAIDS 2017). Approximately, 36.7 million people lived with HIV at the end of 2016 whereas 1.8 million people became infected in 2016 globally (WHO and UNAIDS 2017). The African region is reported to be the most affected with 25.6 million people who lived with HIV in 2016.The region also accounted for almost two thirds of the total global new HIV infections. 
Key populations who were at increased risk of HIV included: men who have sex with other men, people who inject drugs, people in prisons and other closed settings, sex workers and their clients and transgender. According to UNAIDS Report 2017, Kenya had the joint fourth-largest HIV epidemic in the world (alongside Mozambique and Uganda) with 1.6 million people who lived with HIV in 2016. In the same year, 36,000 people died from AIDSrelated illness, while this was still high it has declined steadily from 64,000 in 2010.

One of the key contributors to the spread of HIV is migration of individuals from one region to another. Migration brings more people into close contact and creates a greater mixing of different population groups, that creates ready environment for viral transmission. Through the movement of infected persons, migration also provides a vehicle to transport diseases to places where they have been previously unknown. However, HIV/AIDS require intimate contact for transmission to occur, and migration may help facilitate this by creating a sub-population that is economically, socially, and geographically marginalized. Migration is now a global phenomenon, with 3\% of the world's population living temporarily or permanently outside their country of origin (World Bank 2009). Climate change, urbanization, and expanded trade are likewise driving increased population mobility within and between countries. The relationship between migration and HIV/AIDS was recognized by the United Nations during the General Assembly's Special Session on HIV/AIDS in June 2001. The epidemiology of HIV along transport corridors is not restricted to truck drivers and sex workers. However, lack of effective HIV prevention interventions in spaces of vulnerability along Kenya's major transport corridors is cause for alarm. Long distance truck drivers have been identified as primary dispersers of HIV in sub-Saharan Africa. Truck drivers usually have frequent overnights stays away from home, fewer social controls and excessive waiting periods in ports and border crossings, where the availability of commercial sex increases their vulnerability to infectious diseases and other health problems. Previous reports in India and Kenya found HIV prevalence of $15.9 \%$ and $17.8 \%$ respectively among the long-distance drivers. Condom use among long distance drivers was low and/or inconsistent. Long distance truck drivers are vulnerable to sexually transmitted diseases for several reasons; truck drivers are always on the move, have little or no access to sexual health services, migratory nature of their occupation often disconnect them from their family and community. Truck drivers rarely interact with orthodox practitioners and instead seek help of quacks and home remedies to cure STIs and many lack information about STIs and HIV/AIDS and their prevention. Long-distance truck drivers have robust but diverse sexual cultures. UNAIDS indicated that individuals who lived in extreme mobility; military personnel, truck drivers, among others experienced high vulnerability contexts to infection by HIV (UNAIDS, UNAIDS Gap report 2014). Truck drivers therefore significantly contribute to HIV/AIDS transmission in many countries.

Metapopulation mathematical models have been developed to capture the spatial scale for the spread of disease dynamics. Centre for Media Studies conducted a survey in 2006 among the vulnerable groups; truck drivers were found to be the most potent carriers of HIV due to numerous reasons. The findings proved that $40 \%$ truck drivers were susceptible to have AIDS. Some of the facts collected by CMS are that $95 \%$ of the truckers with AIDS were in the age group of 18 to 45 years and $80 \%$ were married and around $75 \%$ were in the profession for more than five to six years. This showed that more than illiteracy, ignorance and being away from home for long durations were the main reasons behind the spread of the disease among the truckers' community. Rotich et al (2016) studied three levels of disease status in $n$ - patch metapopulation using a simple SIR-HIV epidemic model in a onedimensional nearest neighbor coupling lattice. The basic reproductive ratio $R_{0}(k)$, which is a function of coupling strength $k$, was shown to affect stability characteristics of equilibrium points. The disease-free equilibrium point was proved to be globally asymptotically stable irrespective of the value of $k$ but the stability of the endemic equilibrium point depended on the coupling strength $k$. It was found that at the critical value of coupling strength $k \geq 0.67$, the sub-populations dynamics was synchronized while for $k \leq 0.3$ the subpopulation dynamics was independent. Patch isolation strategy for the control of HIV dispersion required a critical coupling strength of $k_{c} \leq$ 0.15. This interaction restriction reduced $R_{0}$ to values less than one, and the disease was eliminated which made isolation effective. Demographic and epidemiological parameters of Vihiga County in Kenya were used in the study. International Medical Society (2013) analyzed the prevalence of HIV infection and sexual practices among long distance truck drivers who travelled through capital of the Northeast of Brazil. A cross-sectional survey was carried out for five months. Data were collected through application of forms and a rapid -test with blood collection for detection of viral antibodies. It was observed that $100 \%$ of respondents were male, $57.5 \%$ had age between 31 and 50 years, $69 \%$ were married or had a stable relationship and $58.6 \%$ lived in the Northeast. The identified risk factors were: low education (50\%); alcohol use (69.5\%); multiple sexual partners $(50.3 \%)$; lack of use or sporadic use of condoms $(56.3 \%)$. The HIV prevalence detected among truck drivers was $0.8 \%$. The risk factors presented among the truck drivers indicated vulnerability to HIV infection by exposing contamination as well as contributing to spread of the virus in the general population. 
Boahemaa-Atta (2014) investigated the prevalence of HIV, HBV, syphilis and the risk factors among truck drivers using the seaport at Tema. A multivariate analysis was done and the results showed that knowledge of HIV, HBV and syphilis infections and preventive measures were high among the truck drivers. The data indicated a low HIV prevalence among truck drivers in Ghana, however the high prevalence of HBV and syphilis coupled with their risky behavior suggested an increase potential risk of HIV in the truck driving population in Ghana. Mastroberardino (2015) presented a non-linear mathematical model for the transmission dynamics of HIV/AIDS in Cuba. It was assumed that the only mode of transmission is through contact with those yet to be diagnosed with HIV. A linear stability analysis was performed to find the equilibrium of the governing non-linear system. The analysis showed the existence of disease-free and Endemic Equilibrium points and that the stability existence was based on the value of the basic reproduction number. In particular the DFE was locally and globally asymptotically stable for $R_{0}<1$. A unique EE existed whenever $R_{0}>1$, it was locally and globally asymptotically stable whereas the DFE was unstable, and thus the model in consideration does not exhibit a backward bifurcation. Omondi et al (2018) presented a HIV-transmission model with five compartments to describe the trend of HIVinfection within different age groups in Kenya. The model analysis showed that the model has two equilibria, the infection free equilibrium and the endemic equilibrium that are both globally asymptotically stable when the threshold $R_{0}<1$ and $R_{0}>1$, respectively. The model was fitted to data and the HIV trend within different age groups obtained. A sensitivity analysis revealed that the parameters with the most control over the epidemics were treatment rate of HIV patients and the effective contact rate between the susceptible individuals and infected individuals. The results had huge implications to designing policies aimed at reducing new infections especially amongst the young adults. Augustino Isdory (2015) studied the impact of human mobility on HIV transmission in different parts of Kenya. An SIR Meta population model that incorporated the different regions within the country was developed. The model was parameterized using the demographic census data at the time of 2009, HIV data, and mobile phone data adopted to track human mobility. It was found that movement between different regions appears to have relatively small overall effect on the total increase in HIV cases in Kenya. However, the most important consequence of movement patterns was transmission of the disease from high infection to low prevalence areas. Mobility slightly increased high incidence rates in regions with initially low HIV prevalence and slightly decreased incidences in regions with initially high prevalence.

Although previous studies have considered the impact of population mobility on HIV transmission, there is no clear mathematical model that accounts for HIV transmission between geographical regions with different susceptibility and infection rates. This raises an important question, does migrations affects the spread of HIV? The aim of this study was to: develop a deterministic model of HIV transmission between two closed patches that incorporates the Monod Equation in migration of truck drivers being the agents of transmission; compute the basic reproduction number; determine the equilibria points and analyze their stabilities and finally carry out numerical simulations. The model was developed using the population based compartmental structure, the Monod function was used to estimate the effect of the number of sex workers on migrations. The basic reproduction number was determined using the Next Generation Matrix method. Numerical simulations were carried out using the available HIV/AIDS population data and parameter values for Mombasa and Nairobi. Section 2 will be Model formulation, section 3; model analysis, section 4; results and discussions and section 5; conclusion and recommendations.

\subsection{Model formulation}

\subsection{Introduction}

The model is based on the classical SI two-patch spatial structure where the hosts move between the two patches at some rate independent of disease status.

\subsection{Model assumptions}

We assume mass-action mixing and transmission $(\beta S I)$ of the population. The availability of Female Sexual Workers is taken as the limiting factor to migration of truck drivers following the Monod function. The hosts move between the two patches at constant specific migration rate. No vital dynamics of births and natural deaths are considered in the model. Thus, a constant population $S+I+D=N$. The time frame for migration of truck drivers is considered to be short. The trucks entering and leaving are only restricted to the patches. The main feature of the model is that the transmission of HIV is contributed by migration and contact of infected individuals and individuals with AIDS with the susceptible sub-population. The individuals with HIV contribute more to the force of infection than those with AIDS. The infection rates for the two patches is considered to be varying.

\subsection{Model description}

The parameters and variables used in the model are described as shown in Table 1 and 2. 
Table 1. Model Variables

Variables Description

$S_{A}(t) \quad$ The number of susceptible individuals in patch A with time

$I_{A}(\mathrm{t}) \quad$ The number of HIV infectives in patch A with time

$D_{A}(t) \quad$ The number of individuals with full blown AIDS in patch A at time $\mathrm{t}$

$S_{B}(t) \quad$ The number of susceptible individuals in patch B at time t

$I_{B}(t) \quad$ The number of HIV infectives in patch B at time $\mathrm{t}$

$D_{B}(t) \quad$ The number of individuals with full blown AIDS in patch B at time $\mathrm{t}$

Table 2. Model parameters

Parameters Description

$\beta_{1} \quad$ The infection rate of patch $\mathrm{A}$

$\beta_{2} \quad$ The infection rate of patch $\mathrm{B}$

$\lambda_{1} \quad$ Force of infection of patch A

$\lambda_{2} \quad$ Force of infection of patch $\mathrm{B}$

$\gamma \quad$ Progression rate from HIV to AIDS stage

$\delta \quad$ AIDS induced-death rate

$m \quad$ the specific rate of migration of truck drivers between the patches

Z the concentration of resource availability for migration

$k_{z} \quad$ the half-velocity constant

$m_{\max } \quad$ The maximum specific migration rate of truck drivers

\subsection{Model structure}

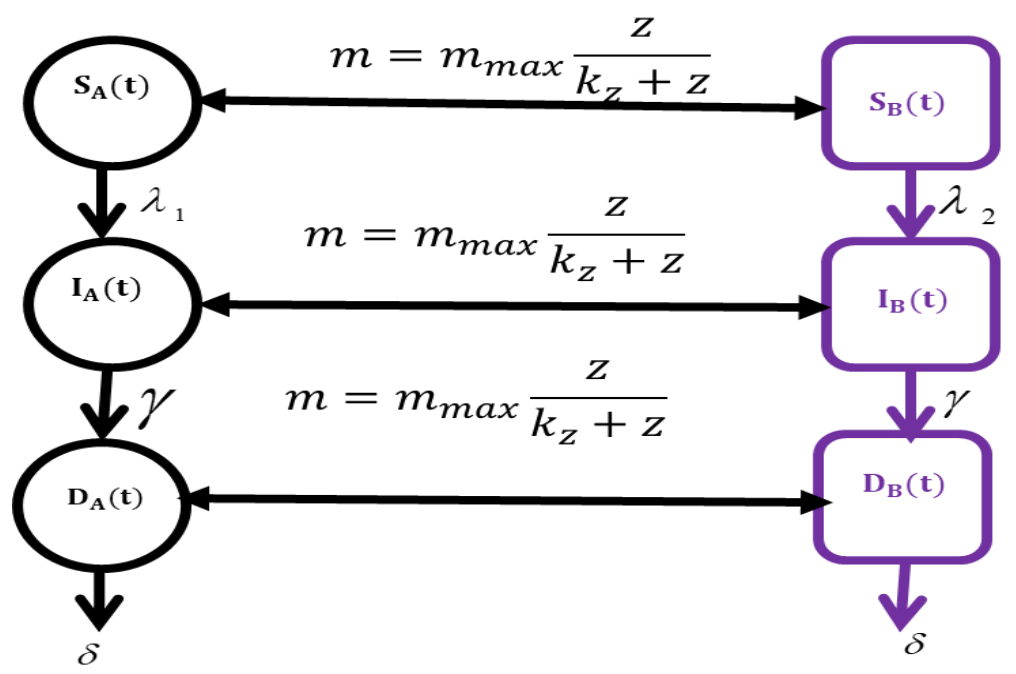

Figure 1. A Two-Patch SI Compartmental Structure 
The following systems of non-linear ordinary differential equations are deduced from Figure 1:

$$
\left.\begin{array}{rl}
\frac{d S_{A}}{d t} & =m\left(S_{B}-S_{A}\right)-\lambda_{1} S_{A} \\
\frac{d I_{A}}{d t} & =m\left(I_{B}-I_{A}\right)+\lambda_{1} S_{A}-\gamma I_{A} \\
\frac{d D_{A}}{d t} & =m\left(D_{B}-D_{A}\right)+\gamma I_{A}-\delta D_{A} \\
\frac{d S_{B}}{d t} & =m\left(S_{A}-S_{B}\right)-\lambda_{2} S_{B} \\
\frac{d I_{B}}{d t} & =m\left(I_{A}-I_{B}\right)+\lambda_{2} S_{B}-\gamma I_{B} \\
\frac{d D_{B}}{d t} & =m\left(D_{A}-D_{B}\right)+\gamma I_{B}-\delta D_{B}
\end{array}\right\}
$$

where

$$
\left.\begin{array}{c}
\lambda_{1}=\beta_{1}\left(I_{A}+\eta_{1} D_{A}\right) \\
\lambda_{2}=\beta_{2}\left(I_{B}+\eta_{2} D_{B}\right) \\
0 \leq \eta_{1}<0.5 \\
0 \leq \eta_{2}<0.5 \\
m=m_{\max } \frac{Z}{k_{Z}+Z}
\end{array}\right\}
$$

The total population size of the patches at time $\mathrm{t}$ is given by;

$$
N(t)=N_{A}(t)+N_{B}(t)=S_{A}(t)+I_{A}(t)+D_{A}(t)+S_{B}(t)+I_{B}(t)+D_{B}(t)
$$

with the initial conditions $S_{A}(0)>0, I_{A}(0)>0, D_{A}(0)>0, S_{B}(0)>0, I_{B}(0)>0$ and $D_{B}>0$.

Thus $\frac{d N}{d t}=\frac{d S_{A}}{d t}+\frac{d I_{A}}{d t}+\frac{d D_{A}}{d t}+\frac{d S_{B}}{d t}+\frac{d I_{B}}{d t}+\frac{d D_{B}}{d t}$

$\frac{d N}{d t}=\frac{d N_{A}}{d t}+\frac{d N_{B}}{d t}$

$$
\frac{d N}{d t}=-\delta\left(\mathrm{D}_{A}+\mathrm{D}_{B}\right)
$$

\section{Model analysis}

The positivity and boundedness of the solutions, DFEP and EEP, basic reproduction number of the patches, and the combined reproduction number are determined as follows;

\subsection{Positivity and Boundedness of the solutions}

\section{Theorem:}

The solutions $\mathrm{S}_{\mathrm{A}}(\mathrm{t}), \mathrm{S}_{\mathrm{B}}(\mathrm{t}), \mathrm{I}_{\mathrm{A}}(\mathrm{t}), \mathrm{I}_{\mathrm{B}}(\mathrm{t}), \mathrm{D}_{\mathrm{A}}(\mathrm{t})$ and $\mathrm{D}_{\mathrm{B}}(\mathrm{t})$ are non-negative for all $\mathrm{t}>0$ and $\lim _{t \rightarrow \infty} N(t)=N(0)$

Proof: Let $\mathrm{S}_{\mathrm{A}}(\mathrm{t}), \mathrm{S}_{\mathrm{B}}(\mathrm{t}), \mathrm{I}_{\mathrm{A}}(\mathrm{t}), \mathrm{I}_{\mathrm{B}}(\mathrm{t}), \mathrm{D}_{\mathrm{A}}(\mathrm{t})$ and $\mathrm{D}_{\mathrm{B}}(\mathrm{t})$ be the solutions of the system (1) with non-negative initial conditions $S_{A}(0) \geq 0, I_{A}(0) \geq 0, D_{A}(0) \geq 0, S_{B}(0) \geq 0, I_{B}(0) \geq 0$ and $D_{B} \geq 0$

Since $\frac{d S_{A}}{d t}=-\lambda_{1} S_{A}+m_{\max } \frac{s}{k_{S}+s}\left(S_{B}-S_{A}\right)$, it follows directly from the first equation of the system (1a) and only taking the negative part that $\frac{d S_{A}}{d t} \geq-\lambda_{1} S_{A}$.Hence $S_{A}(t)=C_{2} e^{-\int_{0}^{t} \lambda_{1}(s) d s}$ 
the integral of any negative exponential function is real and positive. Applying the initial conditions $t=0$ it follows from (7) that $S_{A}(t)=S_{0} e^{-\int_{0}^{t} \lambda_{1}(s) d s} \geq 0$, so $S_{0} e^{-\int_{0}^{t} \lambda_{1}(s) d s}$ is a non-negative function of t, thus $S_{A}(t)$ stays positive. By integration, the positivity of $S_{B}(t), I_{A}(t), I_{B}(t), D_{A}(t)$ and $D_{B}(t)$ are proved similarly along the same lines. From equation (1b) to (1c), it can be shown that:

$$
\begin{aligned}
& I_{A}(t) \geq C_{3} e^{-\gamma t} \geq 0, D_{A}(t) \geq C_{4} e^{-\delta t} \geq 0 ; S_{B}=C_{5} e^{-\int_{0}^{t} \lambda_{2}(s) d s} \geq 0, I_{B}=C_{6} e^{-\gamma t} \geq 0 \text { and } \\
& D_{B}=C_{7} e^{-\delta t} \geq 0
\end{aligned}
$$

Similarly, From (6) $\frac{d N}{d t} \geq-\delta\left(D_{A}+D_{B}\right)$ and on integrating we have $N(t) \geq-\delta \int_{0}^{t}\left(D_{A}(\mathrm{~s})+D_{B}(s)\right) d s$ Thus, $N(t)=C+k e^{-\delta t}+h e^{-\delta t}$ and inserting the initial condition $t=0, N(0)=C+k+h=N_{0}$. Hence $\lim _{t \rightarrow \infty} N(t)=N(0)$.

\subsection{Disease Free -Equilibrium Point (DFEP)}

We determine the DFEP of the system (1). Supposing that the communities in the patches has not experienced HIV infection, then it is expected to remain free of HIV-infection. This implies that the entire community is rendered susceptible to the infection. In this respect, we set the system (1) to zero and there being no infectious individuals, $I_{A}^{0}=D_{A}^{0}=I_{B}^{0}=D_{B}^{0}=0$. From the resulting equations, we obtain $\lambda_{1}=\lambda_{2}=0$, and $S_{A}^{0}=S_{B}^{0}$ implying that the number of susceptible individuals in both patches is constant. Therefore, the system (1) has a disease-free equilibrium point, denoted as $E^{0}=\left(S_{A}^{0}, I_{A}^{0}, D_{A}^{0}, S_{B}^{0}, I_{B}^{0}, D_{B}^{0}\right)=\left\{N_{A}(0), 0,0, N_{B}(0), 0,0\right\}$

\subsection{Basic reproduction number, $\boldsymbol{R}_{\boldsymbol{o}}$}

$R_{o}$ is defined as the number of secondary infections that would occur when a single infectious individual is introduced into an entirely susceptible population, and considered over the lifetime of an episode of the disease (Van den Driessche 2017). It was determined as the spectral radius of the Next Generation Matrix Method $\left(F V^{-1}\right)$.

\subsubsection{Basic reproduction number for patch $A$}

We obtain the Matrix of new infections as: $f_{A}=\left[\begin{array}{c}\beta_{1} S_{A}\left(I_{A}+\eta_{1} D_{A}\right) \\ 0\end{array}\right]$

and matrix of transfer as:

$$
v_{A}=\left[\begin{array}{c}
\gamma I_{A}-m\left(I_{B}-I_{A}\right) \\
-\gamma I_{A}+\delta D_{A}-m\left(D_{B}-D_{A}\right)
\end{array}\right]
$$

The corresponding Jacobian matrix of (9) and (10) are:

$$
\begin{aligned}
& F_{A}=\left[\begin{array}{cc}
\beta_{1} S_{A} & \beta_{1} \eta_{1} S_{A} \\
0 & 0
\end{array}\right] \\
& V_{A}^{-1}=\left\{\left\{\frac{1}{m+\gamma}, 0\right\},\left\{\frac{\gamma}{(m+\gamma)(m+\delta)}, \frac{1}{m+\delta}\right\}\right\}
\end{aligned}
$$

The Eigen values of the matrix $F_{A} V_{A}{ }^{-1}$ at Disease-Free Equilibrium Point are:

$\left\{0, \frac{\mathrm{s}_{A}^{O} \beta_{1}\left(m+\delta+\gamma \eta_{1}\right)}{(m+\gamma)(m+\delta)}\right\}$

Thus, the basic reproduction number for patch $\mathrm{A}$ was obtained as the spectral radius of $F_{A} V_{A}^{-1}$

$$
R_{O}^{A}=\frac{\mathrm{s}_{A}^{O} \beta_{1}\left(m+\delta+\gamma \eta_{1}\right)}{(m+\gamma)(m+\delta)}
$$




\subsubsection{Basic reproduction number for patch $B$}

Similarly, we determine the matrix of new infections as: $f_{B}=\left[\begin{array}{c}\beta_{2} S_{B}\left(I_{B}+\eta_{2} D_{B}\right) \\ 0\end{array}\right]$

and $v_{B}=\left[\begin{array}{c}\gamma I_{B}-m\left(I_{A}-I_{B}\right) \\ -\gamma I_{B}+\delta D_{A}-m\left(D_{A}-D_{B}\right)\end{array}\right]$

The corresponding Jacobian matrix of (16) and (17) for patch B are:

$F_{B}=\left[\begin{array}{cc}\beta_{2} S_{B} & \beta_{2} \eta_{2} S_{B} \\ 0 & 0\end{array}\right]$

$V_{B}^{-1}\left\{\left\{\frac{1}{m+\gamma}, 0\right\},\left\{\frac{\gamma}{(m+\gamma)(m+\delta)}, \frac{1}{m+\delta}\right\}\right\}$

The Eigen values of the Jacobian matrix $F_{B} V_{B}{ }^{-1}$ at Disease-Free Equilibrium Point were obtained as:

$\left\{0, \frac{\mathrm{S}_{B}^{O} \beta_{2}\left(m+\delta+\gamma \eta_{2}\right)}{(m+\gamma)(m+\delta)}\right\}$

Thus, the basic reproduction number for patch $\mathrm{B}$ is obtained as the spectral radius of $F_{B} V_{B}{ }^{-1}$

$R_{O}^{B}=\frac{\mathrm{s}_{B}^{O} \beta_{1}\left(m+\delta+\gamma \eta_{2}\right)}{(m+\gamma)(m+\delta)}$

\subsubsection{The combined basic reproduction number for both patches}

We consider the Jacobian matrix $F_{A B}$ for the entire system and the inverse of the Jacobian matrix as follows

$V_{A B}^{-1}=\left\{\left\{\frac{m+\gamma}{2 m \gamma+\gamma^{2}}, 0, \frac{m}{2 m \gamma+\gamma^{2}}, 0\right\},\left\{\frac{1}{2 \delta}, \frac{1}{2 \delta}, \frac{1}{2 \delta}, \frac{1}{2 \delta}\right\},\left\{\frac{m}{2 m \gamma+\gamma^{2}}, 0, \frac{m+\gamma}{2 m \gamma+\gamma^{2}}, 0\right\},\left\{\frac{1}{2}\left(-\frac{\gamma}{m(2 m+\gamma)}+\frac{1}{\delta}\right), \frac{1}{2}\left(-\frac{1}{m}+\right.\right.\right.$ $\left.\left.\left.\frac{1}{\delta}\right), \frac{1}{2}\left(\frac{\gamma}{m(2 m+\gamma)}+\frac{1}{\delta}\right), \frac{m+\delta}{2 m \delta}\right\}\right\}$

$F_{A B} V_{A B}^{-1}=\left\{\left\{\frac{(m+\gamma) S_{A} \beta_{1}}{2 m \gamma+\gamma^{2}}+\frac{s_{A} \beta_{1} \eta_{1}}{2 \delta}, \frac{s_{A} \beta_{1} \eta_{1}}{2 \delta}, \frac{m s_{A} \beta_{1}}{2 m \gamma+\gamma^{2}}+\frac{s_{A} \beta_{1} \eta_{1}}{2 \delta}, \frac{s_{A} \beta_{1} \eta_{1}}{2 \delta}\right\},\{0,0,0,0\},\left\{\frac{m s_{B} \beta_{2}}{2 m \gamma+\gamma^{2}}+\frac{1}{2}\left(-\frac{\gamma}{m(2 m+\gamma)}+\right.\right.\right.$ $\left.\left.\left.\frac{1}{\delta}\right) S_{B} \beta_{2} \eta_{2}, \frac{1}{2}\left(-\frac{1}{m}+\frac{1}{\delta}\right) S_{B} \beta_{2} \eta_{2}, \frac{(m+\gamma) S_{B} \beta_{2}}{2 m \gamma+\gamma^{2}}+\frac{1}{2}\left(\frac{\gamma}{m(2 m+\gamma)}+\frac{1}{\delta}\right) S_{B} \beta_{2} \eta_{2}, \frac{(m+\delta) S_{B} \beta_{2} \eta_{2}}{2 m \delta}\right\},\{0,0,0,0\}\right\}$

At Disease-Free Equilibrium Point, the matrix $F V^{-1}$ becomes:

$$
\begin{aligned}
& \left\{\left\{\frac{(m+\gamma) \mathrm{S}_{A}^{0} \beta_{1}}{2 m \gamma+\gamma^{2}}+\frac{\mathrm{S}_{A}^{0} \beta_{1} \eta_{1}}{2 \delta}, \frac{\mathrm{S}_{A}^{0} \beta_{1} \eta_{1}}{2 \delta}, \frac{m \mathrm{~S}_{A}^{0} \beta_{1}}{2 m \gamma+\gamma^{2}}+\frac{\mathrm{S}_{A}^{0} \beta_{1} \eta_{1}}{2 \delta}, \frac{\mathrm{s}_{A}^{0} \beta_{1} \eta_{1}}{2 \delta}\right\},\{0,0,0,0\},\left\{\frac{m \mathrm{~S}_{B}^{0} \beta_{2}}{2 m \gamma+\gamma^{2}}+\frac{1}{2}\left(-\frac{\gamma}{m(2 m+\gamma)}+\frac{1}{\delta}\right) \mathrm{S}_{B}^{0} \beta_{2} \eta_{2}, \frac{1}{2}\left(-\frac{1}{m}+\right.\right.\right. \\
& \left.\left.\left.\frac{1}{\delta}\right) \mathrm{S}_{B}^{0} \beta_{2} \eta_{2}, \frac{(m+\gamma) \mathrm{S}_{B}^{0} \beta_{2}}{2 m \gamma+\gamma^{2}}+\frac{1}{2}\left(\frac{\gamma}{m(2 m+\gamma)}+\frac{1}{\delta}\right) \mathrm{S}_{B}^{0} \beta_{2} \eta_{2}, \frac{(m+\delta) \mathrm{S}_{B}^{0} \beta_{2} \eta_{2}}{2 m \delta}\right\},\{0,0,0,0\}\right\}
\end{aligned}
$$

The Eigen values of $F V^{-1}$ is obtained as:

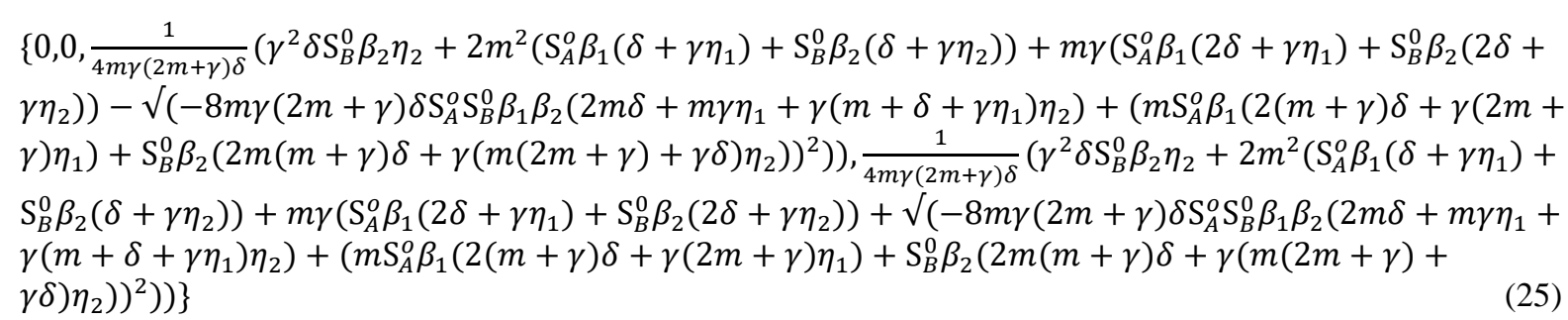

Using the NGM the basic reproduction number is obtained as the spectral radius of $F V^{-1}$ which is:

$$
\begin{gathered}
R_{o}{ }^{A B}=\frac{1}{4 m \gamma(2 m+\gamma) \delta}\left(\gamma^{2} \delta \mathrm{S}_{B}^{0} \beta_{2} \eta_{2}+2 m^{2}\left(\mathrm{~S}_{A}^{o} \beta_{1}\left(\delta+\gamma \eta_{1}\right)+\mathrm{S}_{B}^{0} \beta_{2}\left(\delta+\gamma \eta_{2}\right)\right)+m \gamma\left(\mathrm{S}_{A}^{o} \beta_{1}\left(2 \delta+\gamma \eta_{1}\right)+\right.\right. \\
\left.\mathrm{S}_{B}^{0} \beta_{2}\left(2 \delta+\gamma \eta_{2}\right)\right)+\sqrt{ }\left(-8 m \gamma(2 m+\gamma) \delta \mathrm{S}_{A}^{o} \mathrm{~S}_{B}^{0} \beta_{1} \beta_{2}\left(2 m \delta+m \gamma \eta_{1}+\gamma\left(m+\delta+\gamma \eta_{1}\right) \eta_{2}\right)+\left(m \mathrm{~S}_{A}^{o} \beta_{1}(2(m+\right.\right.
\end{gathered}
$$


$\left.\left.\left.\left.\gamma) \delta+\gamma(2 m+\gamma) \eta_{1}\right)+\mathrm{S}_{B}^{0} \beta_{2}\left(2 m(m+\gamma) \delta+\gamma(m(2 m+\gamma)+\gamma \delta) \eta_{2}\right)\right)^{2}\right)\right)$

From equation (15), (21) and (26), a value of $R_{0}^{A}<1$ implies that each individual is only able to infect less than one individual on average, so that the infection will die out. A value of $R_{0}^{A}>1$ implies that each individual is able to infect more than one individual on average and the infection is expected to persist in the population. Hence, $R_{0}^{A}=1$ is a crucial epidemic threshold.

\subsection{Existence of Endemic Equilibrium Point (EEP)}

Anderson and May (1991), proposed the following property of epidemic models

Property: The disease always dies out and therefore epidemic models don't possess an endemic equilibrium state.

\section{Proof:}

We considered a single patch A and special case in our system where $m=0$.The basic reproduction number for patch A in (15) becomes $R_{O}^{A *}=\frac{\mathrm{s}_{A}^{O} \beta_{1}\left(\delta+\gamma \eta_{1}\right)}{\gamma \delta}$ and our system of equations (1) reduces to:

$$
\left.\begin{array}{c}
\frac{d S_{A}}{d t}=-\beta_{1} S_{A}\left(I_{A}+\eta_{1} D_{A}\right) \\
\frac{d I_{A}}{d t}=\beta_{1} S_{A}\left(I_{A}+\eta_{1} D_{A}\right)-\gamma I_{A} \\
\frac{d D_{A}}{d t}=\gamma I_{A}-\delta D_{A}
\end{array}\right\}
$$

Solving for $S_{A}^{*}, I_{A}^{*}, D_{A}^{*}$ at endemic state and equating the system of equations (27) to zero and from (27c) we have $\gamma I_{A}^{*}=\delta D_{A}^{*}$, which yields $D_{A}^{*}=\frac{\gamma}{\delta} I_{A}^{*}$, substituting $D_{A}^{*}$ into $\beta_{1} S_{A}^{*}\left(I_{A}^{*}+\eta_{1} D_{A}^{*}\right)-\gamma I_{A}^{*}=0$ we obtain $\beta_{1} S_{A}^{*}\left(I_{A}^{*}+\eta_{1} \frac{\gamma}{\delta} I_{A}^{*}\right)-\gamma I_{A}^{*}=0$, factoring $I_{A}^{*}$, we obtain $I_{A}^{*}\left\{\beta_{1} S_{A}^{*}\left(1+\eta_{1} \frac{\gamma}{\delta}\right)-\gamma\right\}=0$.This implies that $I_{A}^{*}=0$ and so $D_{A}^{*}=0$. Similarly, $\beta_{1} S_{A}^{*}\left(1+\eta_{1} \frac{\gamma}{\delta}\right)-\gamma=0$, giving

$$
S_{A}^{*}=\frac{\gamma}{\beta_{1}\left(1+\eta_{1} \frac{\gamma}{\delta}\right)}=\frac{S_{A}^{0}}{R_{A}^{0}}
$$

From the above prove it can be concluded that the infection will die out if $I_{A}^{*}=0$ and $D_{A}^{*}=0$ and that the system does not display an endemic state. This completed the proof of the property proposed by Anderson and May.

In addition, Anderson and May also proposed the theorem for epidemic models as stated below

\section{Theorem 1.}

If $R_{O}^{A B}<1$ the infectious classes decrease with time monotonically to zero as $t \rightarrow \infty$ (at exponential rate).

If $R_{O}^{A B}>1$, then the infectious class starts increasing with time, reaches its maximum, and then decreases to zero as $t \rightarrow \infty$.

If $R_{O}^{A B}=1$, then the infectious classes decrease to zero with time as $t \rightarrow \infty$.

\section{Proof (i)}

We considered a single patch A and special case in our system where $m=0$.The reproduction number for patch A becomes $R_{O}^{A *}=\frac{s_{A}^{O} \beta_{1}\left(\delta+\gamma \eta_{1}\right)}{\gamma \delta}$ and the system of equations (1) reduces to:

$$
\left.\begin{array}{c}
\frac{d S_{A}}{d t}=-\beta_{1} S_{A}\left(I_{A}+\eta_{1} D_{A}\right) \\
\frac{d I_{A}}{d t}=\beta_{1} S_{A}\left(I_{A}+\eta_{1} D_{A}\right)-\gamma I_{A} \\
\frac{d D_{A}}{d t}=\gamma I_{A}-\delta D_{A}
\end{array}\right\}
$$


Substituting $D_{A}^{*}$ at endemic equilibrium state into $\frac{d I_{A}}{d t}$ we obtain $\frac{d I_{A}^{*}}{d t}=\beta_{1} s_{A}^{*}\left(I_{A}^{*}+\eta_{1} \frac{\gamma}{\delta} I_{A}^{*}\right)-\gamma I_{A}^{*}$

Thus, $\beta_{1} s_{A}^{*}\left(I_{A}^{*}+\eta_{1} \frac{\gamma}{\delta} I_{A}^{*}\right)-\gamma I_{A}^{*} \leq I_{A}^{*}\left\{\beta_{1} s_{A}^{0}\left(1+\eta_{1} \frac{\gamma}{\delta}\right)-\gamma\right\}$. We determine the condition necessary and sufficient for $I_{A}^{*}\left\{\beta_{1} s_{A}^{0}\left(1+\eta_{1} \frac{\gamma}{\delta}\right)-\gamma\right\}<0$, this implies that $I_{A}^{*} \gamma\left\{\frac{\mathrm{s}_{A}^{O} \beta_{1}\left(\delta+\gamma \eta_{1}\right)}{\gamma \delta}-1\right\}<0$. Since $I_{A}^{*} \gamma$ cannot be less than zero, therefore $\frac{\mathrm{s}_{A}^{O} \beta_{1}\left(\delta+\gamma \eta_{1}\right)}{\gamma \delta}-1<0$ which implies $R_{O}^{A *}<1$ which completed the prove. Similarly, the same condition can be applied to patch B and the entire system, that is $R_{O}^{B *}<1$ and $R_{O}^{A B}<1$ respectively.

For proof (ii) and (iii), the same procedure in (i) is applied but the conditions are $\frac{\mathrm{s}_{A}^{O} \beta_{1}\left(\delta+\gamma \eta_{1}\right)}{\gamma \delta}-1>0$ and $\frac{\mathrm{s}_{A}^{O} \beta_{1}\left(\delta+\gamma \eta_{1}\right)}{\gamma \delta}-1=0$ respectively. This showed that the conditions necessary and sufficient are as outlined in the theorem as $R_{O}^{A}>1$ and $R_{O}^{A}=1$, and so the entire system, that is $R_{O}^{A B}>1$ and $R_{O}^{A B}=1$.

\subsection{Stability analysis of DFEP}

The local and global stability of the disease-free equilibrium point was analyzed based on the following theorems.

Theorem 2: The DFEP is locally asymptotically stable whenever $R_{O}^{A}<1$ and unstable otherwise.

\section{Proof:}

We considered patch A, with the following systems of equations

$$
\left.\begin{array}{rl}
\frac{d S_{A}}{d t} & =m\left(S_{B}-S_{A}\right)-\beta_{1} S_{A}\left(I_{A}+\eta_{1} D_{A}\right) \\
\frac{d I_{A}}{d t} & =m\left(I_{B}-I_{A}\right)+\lambda_{1} S_{A}-\gamma I_{A} \\
\frac{d D_{A}}{d t} & =m\left(D_{B}-D_{A}\right)+\gamma I_{A}-\delta D_{A}
\end{array}\right\}
$$

The local stability of patch A is determined by the signs of the Eigen values of the Jacobean matrix at DFEP. When all the Eigenvalues are negative the DFEP is locally asymptotically stable and unstable otherwise. Determining the Jacobean matrix of the system of equations (5) at DFEP, we obtain:

$$
\left(\begin{array}{ccc}
-m & -\beta_{1} S_{A}^{0} & -\beta_{1} \eta_{1} S_{A}^{0} \\
0 & \beta_{1} S_{A}^{0}-\gamma-m & \beta_{1} \eta_{1} S_{A}^{0} \\
0 & \gamma & -\delta-m
\end{array}\right)
$$

The eigenvalues of the above matrix are:

$$
\left\{\begin{array}{l}
-m, \\
\frac{1}{2}\left(-2 m-\gamma-\delta+S_{A} \beta_{1}-\sqrt{\gamma^{2}-2 \gamma \delta+\delta^{2}-2 \gamma S_{A} \beta_{1}+2 \delta S_{A} \beta_{1}+S_{A}^{2} \beta_{1}^{2}+4 \gamma S_{A} \beta_{1} \eta_{1}}\right), \\
\frac{1}{2}\left(-2 m-\gamma-\delta+S_{A} \beta_{1}+\sqrt{\gamma^{2}-2 \gamma \delta+\delta^{2}-2 \gamma S_{A} \beta_{1}+2 \delta S_{A} \beta_{1}+S_{A}^{2} \beta_{1}^{2}+4 \gamma S_{A} \beta_{1} \eta_{1}}\right)
\end{array}\right\}
$$

It's evident from (33) that the first two eigenvalues are negative. We want to establish the necessary and sufficient condition for $\frac{1}{2}\left(-2 m-\gamma-\delta+S_{A} \beta_{1}+\sqrt{\gamma^{2}-2 \gamma \delta+\delta^{2}-2 \gamma S_{A} \beta_{1}+2 \delta S_{A} \beta_{1}+S_{A}^{2} \beta_{1}^{2}+4 \gamma S_{A} \beta_{1} \eta_{1}}\right)$ to be negative.

Proof: We let $m=0$, then $R_{O}^{A *}=\frac{\mathrm{s}_{A}^{O} \beta_{1}\left(\delta+\gamma \eta_{1}\right)}{\gamma \delta}$.

$$
\begin{aligned}
& \frac{1}{2}\left(-\gamma-\delta+S_{A} \beta_{1}+\sqrt{\gamma^{2}-2 \gamma \delta+\delta^{2}-2 \gamma S_{A} \beta_{1}+2 \delta S_{A} \beta_{1}+S_{A}^{2} \beta_{1}^{2}+4 \gamma S_{A} \beta_{1} \eta_{1}}\right)<0 \\
& \left(\gamma+\delta-S_{A} \beta_{1}\right)>\sqrt{\gamma^{2}-2 \gamma \delta+\delta^{2}-2 \gamma S_{A} \beta_{1}+2 \delta S_{A} \beta_{1}+S_{A}^{2} \beta_{1}^{2}+4 \gamma S_{A} \beta_{1} \eta_{1}} \\
& \left(\gamma+\delta-S_{A} \beta_{1}\right)^{2}>\gamma^{2}-2 \gamma \delta+\delta^{2}-2 \gamma S_{A} \beta_{1}+2 \delta S_{A} \beta_{1}+S_{A}^{2} \beta_{1}^{2}+4 \gamma S_{A} \beta_{1} \eta_{1} \\
& \gamma \delta\left\{1-\frac{S_{A} \beta_{1}\left(\delta+\gamma \eta_{1}\right)}{\gamma \delta}\right\}>0 \text { that is } \gamma \delta\left\{1-R_{O}^{A *}\right\}>0 \text { since } \gamma \delta>0, \text { then } 1-R_{O}^{A *}>0, \text { hence } R_{O}^{A *}<1 .
\end{aligned}
$$


This completed the proof. The same theorem can also be proved for patch B as $R_{O}^{B}<1$ and entire system as $R_{O}^{A B}<$ 1.

Theorem 3: The Disease-Free Equilibrium Point is globally asymptotically stable in Lyapunov sense whenever $\mathrm{R}_{0}^{A B}<1$ and unstable otherwise.

Proof: We considered a special case where $m=0$ of the model in patch A and to proof $\mathrm{R}_{0}^{A B}<1$, we proposed a logarithmic Lyapunov function given as:

$L\left(S_{A}, I_{A}, D_{A}\right)=S_{A}-S_{A}^{0}-S_{A}^{0} \ln \frac{S_{A}}{S_{A}^{0}}+x_{1} I_{A}+x_{2} D_{A}$

where $x_{1}$ and $x_{2}$ are non-negative Lyapunov constants. A well-posed Lyapunov function, $L$ Satisfies the following conditions to ascertain global asymptotic stability:

i) $\quad L\left(S_{A}^{0}, I_{A}^{0}, D_{A}^{0}\right)=0$ and $L\left(S_{A}, I_{A}, D_{A}\right)>0, \forall S_{A}, I_{A}, D_{A} \neq 0$

ii) $\quad \frac{d L\left(S_{A}^{0}, I_{A}^{0}, D_{A}^{0}\right)}{d t}=0$ and $\frac{d L\left(S_{A}, I_{A}, D_{A}\right)}{d t}<0$

Explicitly in (35i) above, $L\left(S_{A}^{0}, I_{A}^{0}, D_{A}^{0}\right)=S_{A}^{0}-S_{A}^{0}-S_{A}^{0} \ln \frac{S_{A}^{0}}{S_{A}^{0}}+x_{1} I_{A}^{0}+x_{2} D_{A}^{0}=0$ since $D_{A}^{0}=I_{A}^{0}=0$ and $\ln 1=$ 0 in the same sense $S_{A} \neq S_{A}^{0}, I_{A} \neq 0, D_{A} \neq 0$ and $\ln \frac{S_{A}}{S_{A}^{0}} \neq 0$ hence $L\left(S_{A}, I_{A}, D_{A}\right)>0$.

In (ii) $\frac{d L\left(S_{A}^{0}, I_{A}^{0}, D_{A}^{0}\right)}{d t}=\left(1-\frac{S_{A}^{0}}{S_{A}^{0}}\right) \frac{d S_{A}^{0}}{d t}+x_{1} \frac{d I_{A}^{0}}{d t}+x_{2} \frac{d D_{A}^{0}}{d t}=0$ since $D_{A}^{0}=I_{A}^{0}=0$ and $1-\frac{s_{A}^{0}}{S_{A}^{0}}=0$. To conclude that the system is globally asymptotically stable, we are only left to prove that $\frac{d L\left(S_{A}, I_{A}, D_{A}\right)}{d t}<0$

The time derivative of the Lyapunov function in (34) is given as:

$\frac{d L\left(S_{A}, I_{A}, D_{A}\right)}{d t}=\left(1-\frac{S_{A}^{0}}{S_{A}}\right) \frac{d S_{A}}{d t}+x_{1} \frac{d I_{A}}{d t}+x_{2} \frac{d D_{A}}{d t}$

Substituting for $\frac{d S_{A}}{d t}, \frac{d I_{A}}{d t}$ and $\frac{d D_{A}}{d t}$ into (36) we have:

$\frac{d L\left(S_{A}, I_{A}, D_{A}\right)}{d t}=\left(1-\frac{S_{A}^{0}}{S_{A}}\right)\left\{-\beta_{1} S_{A}\left(I_{A}+\eta_{1} D_{A}\right)\right\}+x_{1}\left\{\beta_{1} S_{A}\left(I_{A}+\eta_{1} D_{A}\right)-\gamma I_{A}\right\}+x_{2}\left\{\gamma I_{A}-\delta D_{A}\right\}$

$\frac{d L\left(S_{A}, I_{A}, D_{A}\right)}{d t}=S_{A} I_{A}\left(-\beta_{1}+x_{1} \beta_{1}\right)+S_{A} D_{A}\left(-\beta_{1} \eta_{1}+x_{1} \beta_{1} \eta_{1}\right)+I_{A}\left(S_{A}^{0} \beta_{1}-x_{1} \gamma+x_{2} \gamma\right)+D_{A}\left(S_{A}^{0} \beta_{1} \eta_{1}-x_{2} \delta\right)$

Setting $S_{A} I_{A}$ and $D_{A}$ to zero yields, $-\beta_{1}+x_{1} \beta_{1}=0$ and $S_{A}^{0} \beta_{1} \eta_{1}-x_{2} \delta=0$. Solving the equations, we obtain $x_{1}=1$ and $x_{2}=\frac{s_{A}^{0} \beta_{1} \eta_{1}}{\delta}$. Replacing for $x_{1}$ and $x_{2}$ in $\frac{d L\left(S_{A}, I_{A}, D_{A}\right)}{d t}$ we obtain:

$\frac{d L\left(S_{A}, I_{A}, D_{A}\right)}{d t}=I_{A}\left(S_{A}^{0} \beta_{1}-\gamma+\gamma \frac{S_{A}^{0} \beta_{1} \eta_{1}}{\delta}\right)=I_{A} \gamma\left\{\frac{\mathrm{s}_{A}^{O} \beta_{1}\left(\delta+\gamma \eta_{1}\right)}{\gamma \delta}-1\right\}=I_{A} \gamma\left(\mathrm{R}_{0}^{A *}-1\right)$

The necessary and sufficient condition for $\frac{d L\left(S_{A}, I_{A}, D_{A}\right)}{d t}<0$ is $I_{A} \gamma\left(\mathrm{R}_{0}^{A *}-1\right)<0$ which implies that $\mathrm{R}_{0}^{A *}<1$ since $I_{A} \gamma \neq 0$. The same condition can also be demonstrated in patch $\mathrm{B}$ and the entire system and so the necessary and sufficient condition for globally asymptotically stable of the entire system is $\mathrm{R}_{0}^{A B}<1$. This completed the proof.

\section{Results and discussions}

We considered the migrations between two major cities in Kenya; Nairobi and Mombasa. In the model $N_{A}$ and $N_{B}$ represents the total population for Mombasa and Nairobi respectively, whose data was obtained from Kenya National Bureau of Stastitics 2015 as tabulated below. According to Business daily 2019, 3000 truck drivers enters and leaves Mombasa daily. Model variables and parameter values are obtained from the available relevant literature on HIV epidemic models. A summary of the model variables, parameters and their respective values and sources are shown in Table 3. 
Table 3. Initial Conditions

\begin{tabular}{|c|c|c|c|c|c|}
\hline Parameter & Value & Source & $\begin{array}{l}\text { Initial } \\
\text { conditions }\end{array}$ & Value & Source \\
\hline$\beta_{1}$ & $0.002397 /$ year & $\begin{array}{l}\text { Kenya HIV } \\
\text { county profiles } \\
2016\end{array}$ & $N_{A}$ & $1,145,259$ & $\begin{array}{l}\text { KNBS } \\
\text { population } \\
\text { projection } \\
(2015)\end{array}$ \\
\hline$\beta_{2}$ & $0.001177 /$ year & $\begin{array}{l}\text { Kenya HIV } \\
\text { county profiles } \\
2016\end{array}$ & $N_{B}$ & $4,232,087$ & $\begin{array}{l}\text { KNBS } \\
\text { population } \\
\text { projection } \\
(2015)\end{array}$ \\
\hline$\gamma$ & $0.125 /$ year & $\begin{array}{l}\text { Baryarama F, } \\
\text { Mugisha JYT, } \\
\text { Luboobi LS., } \\
(2006)\end{array}$ & $S_{A}(0)$ & 1059365 & $\begin{array}{l}\text { KNBS } \\
\text { population } \\
\text { projection } \\
(2015)\end{array}$ \\
\hline$m$ & $\begin{array}{l}0.0007089 \text { to } \\
0.01048 / \text { day }\end{array}$ & $\begin{array}{l}\text { (Business daily, } \\
\text { 2019) }\end{array}$ & $S_{B}(0)$ & 3965466 & $\begin{array}{l}\text { KNBS } \\
\text { population } \\
\text { projection } \\
(2015)\end{array}$ \\
\hline$\delta$ & 0.004326 & $\begin{array}{l}\text { Kenya HIV } \\
\text { county profiles } \\
2016\end{array}$ & $I_{A}(0)$ & 54670 & $\begin{array}{l}\text { Kenya HIV } \\
\text { estimates } \\
\text {,2015 }\end{array}$ \\
\hline$I_{B}(0)$ & 177552 & $\begin{array}{l}\text { Kenya HIV } \\
\text { estimates } 2015\end{array}$ & $D_{A}(0)$ & 31224 & $\begin{array}{l}\text { Kenya HIV } \\
\text { estimates } \\
2015\end{array}$ \\
\hline$D_{B}(0)$ & 80,605 & $\begin{array}{l}\text { Kenya HIV } \\
\text { estimates (2015) }\end{array}$ & & & \\
\hline
\end{tabular}

4.1. Numerical values for model reproduction numbers

On substituting the initial conditions and parameters into (15), (21) and (26), the total secondary infections for Mombasa, Nairobi and combined populations were obtained as:

$R_{O}^{\text {Mombasa }}=16993.69466724191, R_{O}^{\text {Nairobi }}=8383.60474035797, R_{O}^{\text {Mombasa }- \text { Nairobi }}=$ 70614.8740035696

From these results it is observed that $R_{O}^{\text {Mombasa-Nairobi }}>R_{O}^{\text {Mombasa }}>R_{O}^{\text {Nairobi }}$, implying that the migration between Mombasa and Nairobi contributes more to the spread of HIV infection. Despite the fact that Mombasa has a lower population than Nairobi, the rate of spread of HIV infection in Mombasa is more than that of Nairobi. Thus, Nairobi has a higher infection rate than Mombasa.

\subsection{Normalized sensitivity analysis of basic reproduction numbers}

The normalized forward sensitivity index of a variable, $\varnothing$ that depends differentiability on a parameter, $\bar{\vartheta}$, is defined as: $R_{\vartheta}^{\emptyset}=\frac{\partial \emptyset}{\partial \bar{\vartheta}} \times \frac{\vartheta}{\emptyset}($ Ngari \& Koech 2017$)$

On determining the sensitivity indices for all the parameters at DFEP in expressions $R_{O}^{A}, R_{O}^{B}$, and $R_{O}^{A B}$ and substituting their values in the Table 4 we obtain: 
Table 4. Sensitivity Indices

\begin{tabular}{|l|c|c|c|c|c|c|c|}
\hline Parameters & $\beta_{1}$ & $\beta_{2}$ & $\eta_{1}$ & $\eta_{2}$ & $m$ & $\delta$ & $\gamma$ \\
\hline $\begin{array}{l}\text { Sensitivity } \\
\text { index, } R_{O}^{\text {Mombasa }}\end{array}$ & 1 & 0 & 0.00147801 & 0 & $\begin{array}{c}- \\
0.199766\end{array}$ & 0.00145617 & 0.798778 \\
\hline $\begin{array}{l}\text { Sensitivity } \\
\text { index, } R_{O}^{\text {Nairobi }}\end{array}$ & 0 & 1 & 0 & 0.002068 & $\begin{array}{c}- \\
0.199774\end{array}$ & 0.00203743 & 0.798188 \\
\hline $\begin{array}{l}\text { Sensitivity } \\
\text { index, } R_{O}^{\text {Mombasa-Nairobi }}\end{array}$ & 0.05109 & 0.948908 & 0.000137533 & 0.0404551 & $\begin{array}{c}- \\
-\end{array}$ & $\begin{array}{c}- \\
-\end{array}$ & $\begin{array}{c}- \\
0\end{array}$ \\
\hline
\end{tabular}

From the above sensitivity analysis, it is observed that the rate of migrations is inversely proportional to the basic reproduction number while the infection rates is directly proportional to the basic reproduction number. This implies that to reduce the spread of HIV infection more efforts and measures should be focused in reducing the infections and encouraging more migrations from Mombasa to Nairobi. In addition, all efforts in attempt to reduce the transmission of HIV should be geared towards reducing infection rates in Nairobi than in Mombasa.

\subsection{Numerical simulations}

Numerical simulation was carried out using fourth order Runge-Kutta method in MatlabR2017a to assess the dynamical behavior of the system of non-linear ordinary differential equations presented in the presence of control parameters. The computer simulations are performed using the initial conditions and parameters in Table 4 and presented graphically as shown in Figure (2) to (16)

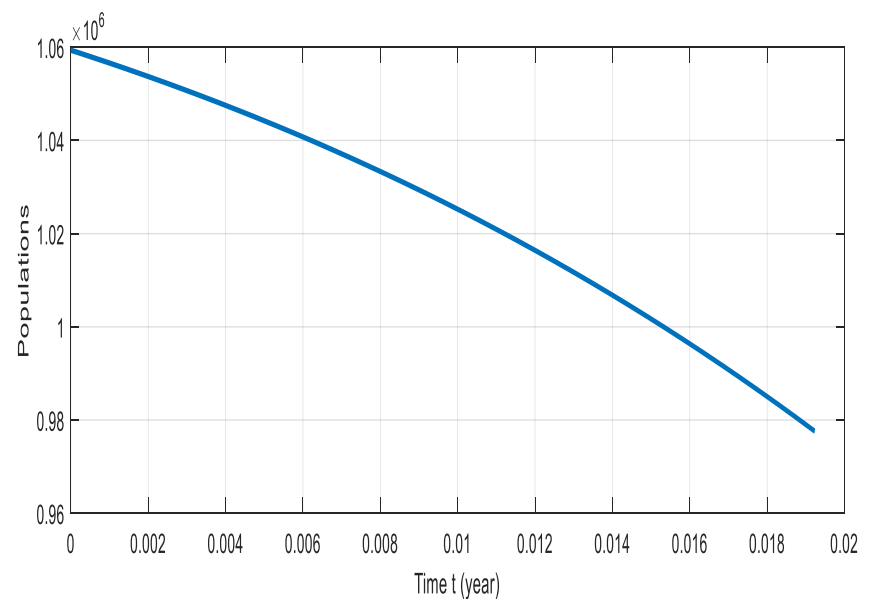

Figure 2. Population of susceptible individuals in Mombasa with time

The population of susceptible individuals decreases rapidly with time. This is because the susceptible individuals get infected with time and the infected individuals move to the next class of infectious individuals since there are no births. At the same time a proportion of the susceptible individuals move to Mombasa thus the decrease. 


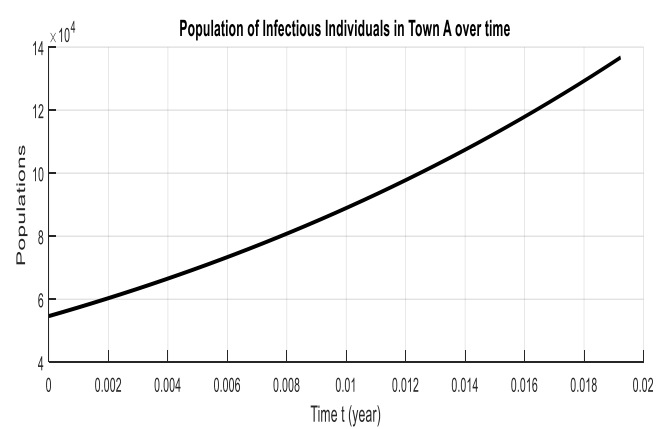

Figure 3 . Population of infectious individuals in Mombasa with time

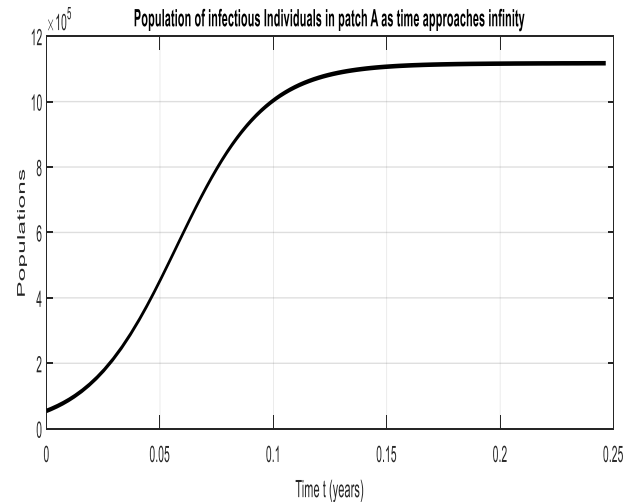

Figure 4. Population of infectious individuals in

Mombasa as time approaches to infinity

Initially, the population of infectious individuals in patch A increases with time when $R_{0}^{A B}>1$. However, as time approaches to infinity, the population of infectious individuals in patch A remains constant after attaining a maximum value. This confirmed the theorem proposed by Anderson and May.

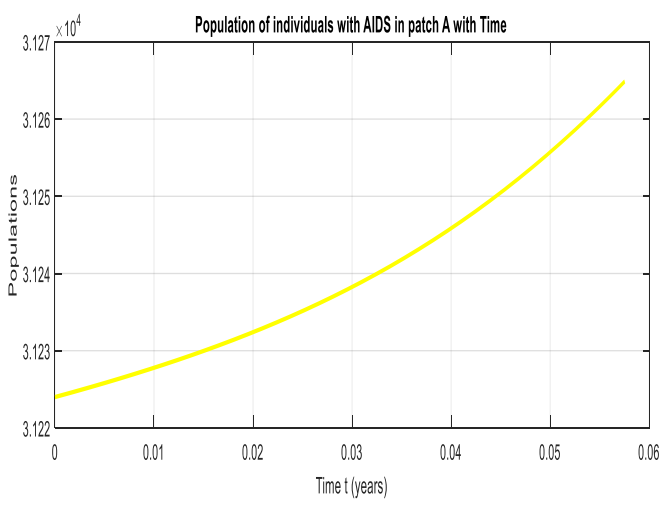

Figure 5. Population of AIDS individuals in Mombasa with time

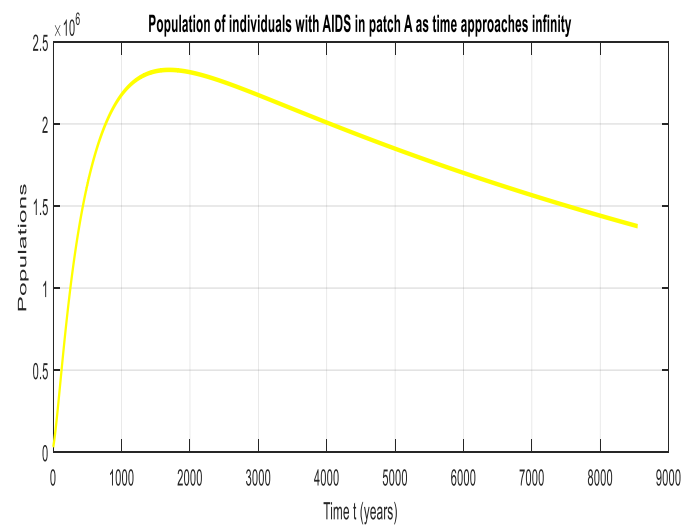

Figure 6. Population of AIDS individuals in

Mombasa as time approaches to infinity

Initially, the population of people at the final stage of AIDS increases with time. However, as time approaches to infinity, the population tends to reduce to zero.

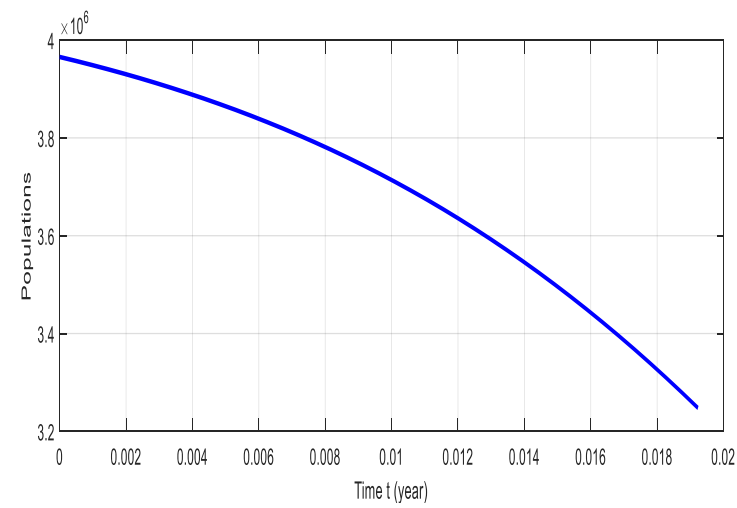

Figure 7. Population of susceptible individuals in Nairobi with Time 
The population of susceptible in Nairobi decreases rapidly with time and tends to zero with time when $R_{0}^{A B}>$ 1.This is because there are no births as well as transition of infected individuals to the next compartment at the same time migration of susceptible individuals to Mombasa.

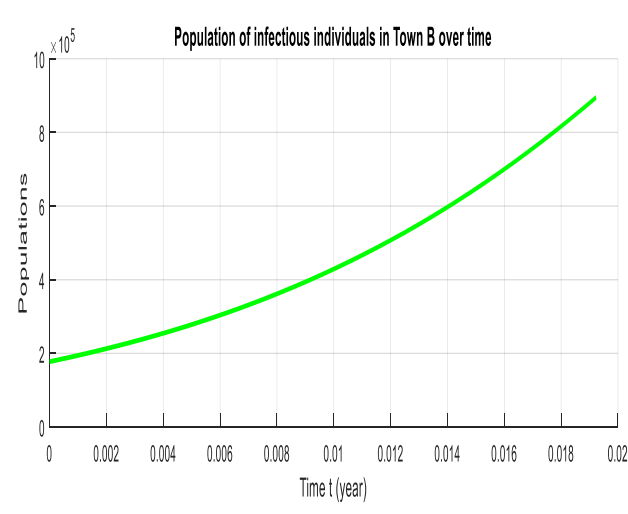

Figure 8 . Population of infectious individuals in Nairobi with time

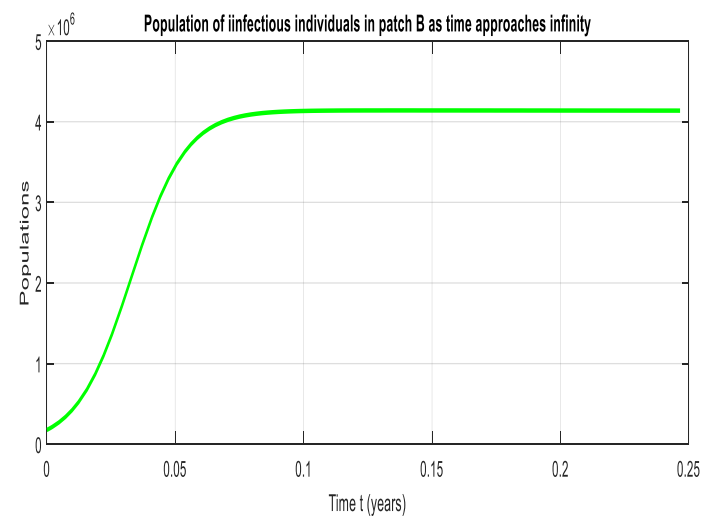

Figure 9. Population of infectious individuals in Nairobi as time approaches to infinity

As susceptible individuals become infected, the population of infectious individuals in Nairobi increases with time when $R_{0}^{A B}>$ 1.A proportion of infected individuals from Mombasa also attributes to this increase. However, as time approaches to infinity, the population reaches a maximum point and then remains constant throughout the infection period. This confirmed the theorem proposed by Anderson and May.

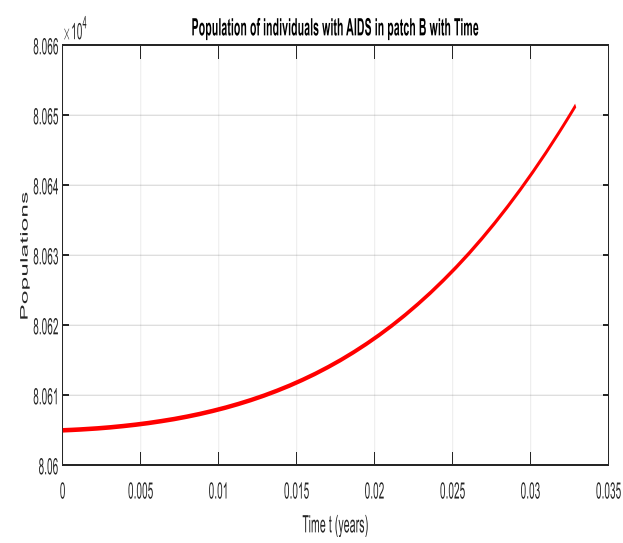

Figure 10. Population of individuals with AIDS in Nairobi with time

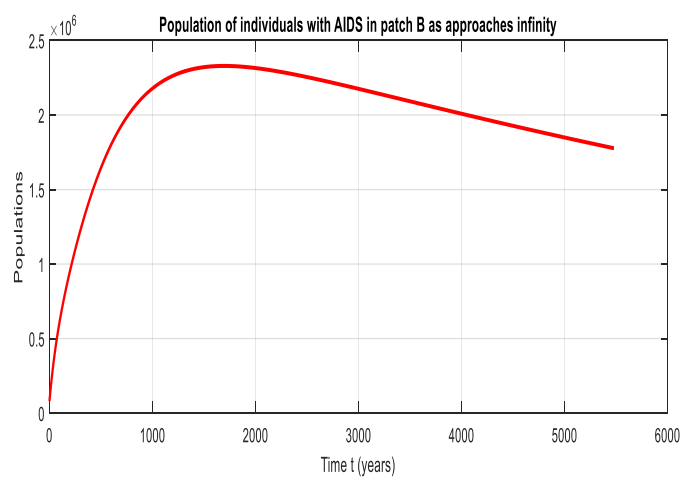

Figure 11. Population of individuals in Nairobi as time approaches infinity

Initially, the population of individuals with AIDS in Nairobi increases gradually with time when $R_{0}^{A B}>1$.This is due to the infected individuals progressing to AIDS class and addition of AIDS patients from Mombasa. However, as time approaches infinity, at first the population increases rapidly before it reaches a maximum value and then decreases slowly to zero with time. This agrees with the theorem proposed by Anderson and May.

\subsection{Effect of Migration parameter, $\boldsymbol{m}$ on Female Sex Workers, $Z$}

The migration parameter follows the Monod function, $m=m_{\max } \frac{Z}{k_{Z}+Z}$ where $K_{z}=0.25$ from the experimental data for bacterial growth by Smith. In our model we use $z$ as the Female Sex Workers. From table 3, the maximum migration rate is 0.01048 /day, thus the modified Monod equation becomes, $m=0.01048 \frac{Z}{(0.25+Z)}$. The minimum and maximum estimated values for FSW in Mombasa were taken as 10,000 and 100,000 respectively (Onyulo 
2016). The estimated minimum number of Female Sex Workers was taken as 2400 per night (Wang 2015). The maximum value may be obtained for a period of three weeks or a month.

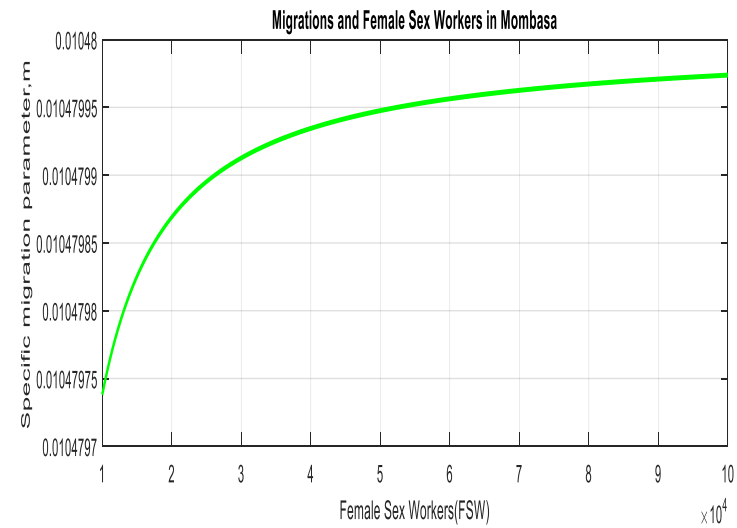

Figure 12. Migration and Female Sex Workers in

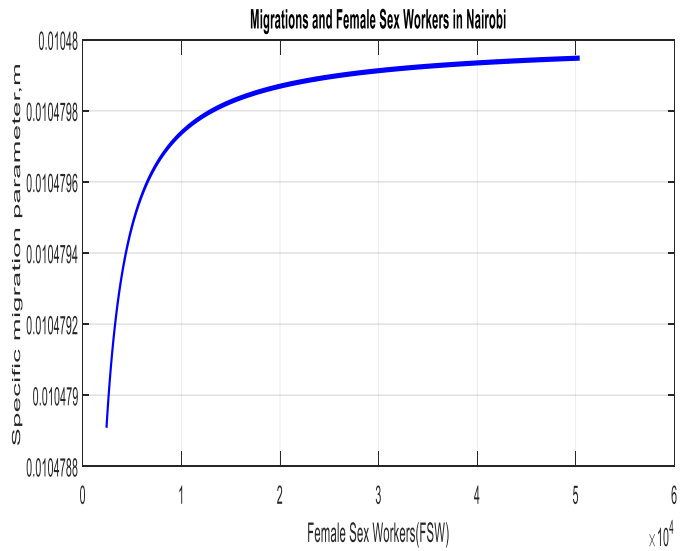

Figure 13. Migration and Female Sex Workers in

Mombasa

Nairobi

From the above curves, the rate of migration increases slowly with increase in the number of FSW in Mombasa and rapidly increases with increase in the number of FSW, until a maximum value is reached for both towns. However, the saturation point is realized much earlier in Nairobi than in Mombasa.

\subsection{Effect of migration parameter, $m$ on the basic reproduction numbers}

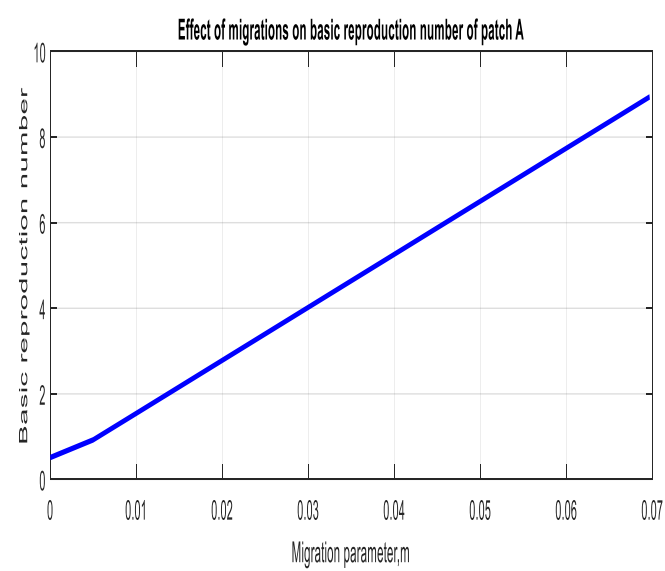

Figure 14. Basic reproduction for Mombasa and migration

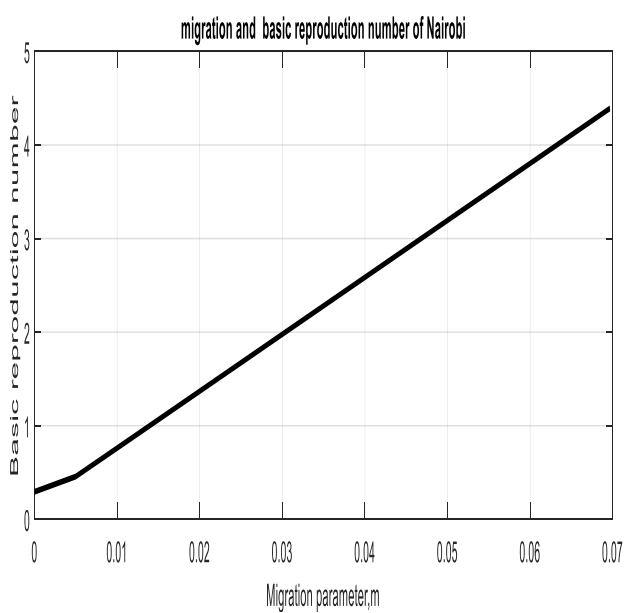

Figure15.Basic reproduction for Nairobi and migration

Initially, the basic reproduction number increases slightly with increase in the migration parameter due to lower infection rate and then increases rapidly from 0.005 to the maximum migration parameter due to higher infection rate in Mombasa. Similarly, this is the case in Nairobi but at a slower rate due to lower infection rate. 


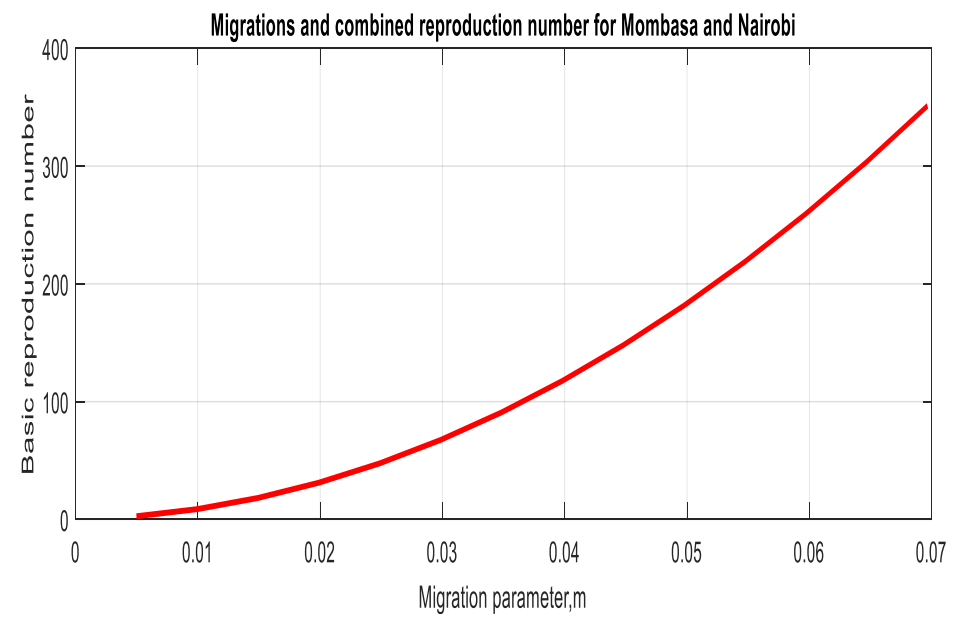

Figure 16: Migrations on combined reproduction number for Mombasa and Nairobi

From 0.005 to 0.02 , the rate of migration with combined basic reproduction number for the model is slow (lag stage) and then increases rapidly to the maximum rate. The minimum and maximum value for the migration parameter is tabulated in Table 3 .

\section{Conclusion and recommendation \\ 5.1. Conclusion}

In this study, HIV transmission model through two closed patches in migration is derived and analyzed. The basic reproduction number $R_{o}$ for the patches was determined as the dominant eigenvalue of the Jacobian matrix using the NGM. The DFEP was also obtained. The model did not show the existence of the endemic equilibrium point. The DFEP was proved to be locally asymptotically stable whenever $R_{0}^{A B}<1$.A suitable Lyapunov function was constructed and the analysis showed that the disease-free equilibrium point of the model was globally asymptotically stable whenever $R_{0}^{A B}<1$. Normalized sensitivity indices of basic reproduction number with respect to control parameters were obtained as outlined in Table 4. From the analysis it was observed that the rate of migration is inversely proportional to $R_{0}^{A B}$ while the infection rate is directly proportional to $R_{0}^{A B}$. This implies that to reduce the spread of HIV infection more efforts and measures should be focused in reducing the infections and encouraging more migrations from Mombasa with higher infection rate to Nairobi with lower infection rate. In addition, all efforts in attempt to reduce the transmission of HIV should be geared towards reducing infection rates in Nairobi than Mombasa. The Monod curves showed that migration increases with increase in the model reproduction number and the number of female sex workers.

\subsection{Recommendations}

From the research findings it is that recommended health practitioners, government policy makers and institutions of supply chain management should make deliberate efforts in regulating the migration of individuals to regions or transport routes used by truckers. The health implications of this observation are that keeping the reproduction number below unity may be necessary in reducing the spread of HIV infection by truck drivers. Health care facilities and services should also be provided along the transport corridors to mitigate HIV transmission. Further research work should focus on incorporating vital dynamics, increase the patch size as well as varying the migration parameter.

\section{References}

Anderson,R.M. and May,R.M., 1991. Infectious Diseases of Humans: Dynamics and Control. Bulletin of Mathematical Biology. 
Avert, 2019. avert.org. [Online]

Available at: https://www.avert.org

Boahemaa-Atta, P., 2014. Prevalence of HIV,Syphilis,Hepatitis B,and Risk factors among the Truck drivers in Tema,Ghana..

Business daily, A., 2019. Mombasa plans parking yards for trucks to ease.

IOM, 2010a. An anlysis of Migration Health in Kenya, s.1.: Regional Mission for East \& Central Africa.

KNBS, 2015. Population projections, Nairobi-Kenya: KNBS.

Ministry of Health, K., 2008. Kenya ,AIDS indicator Survey 2007, Nairobi-Kenya: National AIDS and STI Control Programme(NASCOP).

Ngari, C.G. and Koech,W., 2017. Modeling kenya's domestic radicalization like a disease by incorporating efforts of clergies, rehabilitation centers and justice system. Journal of Applied Sciences, Engineering and Technology for Development(JASETD), 2,(1,), pp. 1-33.

Omondi,E.O,Mbogo,R. and Luboobi,L.S, 2018. Mathematical Modeling of the Impact of testing treatment and control of HIV transmission in Kenya.

Onyulo, T., 2016. Commercial Sex workers, S.L.: Public Radio International (PRI).

Smith, H., n.d. In: Bacterial growth. S.L.:S.N., pp. 1-14.

Statistics, K. N. B. o., 2015. Population projections, s.1.: KNBS.

UNAIDS, 2014. UNAIDS Gap report, S.L.: S.N.

UNAIDS, 2017. UNAIDS Report, S.L.: S.N.

UNAIDS, 2018. [Online]

Available at: $\underline{w w w . u n a i d s . o r g}$

Van den Driessche, P., 2017. Reproduction numbers of infectious disease models. science direct, 2(3), pp. 288303.

Wang, J., 2015. Commercial Sex Workers In Nairobi, S.L.: Duke Global Health Institute.

WHO and UNAIDS, 2017. WHO and UNAIDS report, S.L.: S.N. 
WHO, 2016. Global Information and Education on HIV and AIDS , S.L.: S.N. 